\title{
REVISÃO/REVIEW
}

\section{USO DE BETA-BLOQUEADORES EM PACIENTES MAIORES QUE 65 ANOS COM INSUFICIÊNCIA CARDÍACA SISTÓLICA}

\section{USE OF BETA-BLOCKERS IN PATIENTS OLDER THAN 65 YEARS WITH SYSTOLIC HEART FAILURE}

Lídia Zytynsky Moura1, Solena Ziemer Kusma², Isabela C. B. Garcia3, Maria Eduarda I. A. Da Rocha Loures³, Mariane Adelle P. de Souza ${ }^{3}$

\section{RESUMO}

Esse artigo tem como objetivo apresentar uma revisão de literatura sobre o uso de beta-bloqueadores em pacientes idosos com insuficiência cardíaca sistólica, sua prescrição e eficácia terapêutica. A Insuficiência Cardíaca (IC) é uma síndrome multifatorial de alta incidência, com disfunção ventricular esquerda sistólica na maioria dos casos. A sua prevalência aumenta com a idade e com o número de comorbidades associadas. As diretrizes preconizam como tratamento a associação de um inibidor da enzima conversora da angiotensina II (iECA) ou bloqueador do receptor de angiotensina II (BRA) com beta-bloqueadores (BB), sendo estas drogas disruptivas no tratamento dessa doença.

Descritores: Insuficiência Cardíaca Sistólica. Idosos. Beta-Bloqueadores.

\section{ABSTRACT}

This article aims to present a literature review on the use, prescription and therapeutic efficacy of beta-blockers in elderly patients with systolic heart failure. Heart failure (HF) is a high incidence multifactorial syndrome, with left ventricular systolic dysfunction in most cases. It's prevalence increases with aging and associated comorbidities. The guidelines for the treatment recommend the combination of an angiotensin converting enzyme inhibitor (ACE inhibitor) or angiotensin II receptor blockers (ARB) with beta-blockers (BB), which are disruptive drugs at this ailment's treatment.

Keywords: Systolic Heart Failure. Seniors. Beta-Blockers.

1- Professora da Disciplina de Cardiologia da Pontifícia Universidade Católica do Paraná, Curitiba, Paraná.

2- Professora da Disciplina de Metodologia da Pesquisa da Pontifícia Universidade Católica do Paraná, Curitiba, Paraná.

3- Acadêmica da Escola de Medicina da Pontifícia Universidade Católica do Paraná, Curitiba, Paraná

Contato do Autor / Mail to:

Mariane Adelle P. de Souza - mariane.adelle@hotmail.com

Rua Imaculada Conceição, Número: 1155, Bairro Prado Velho, Curitiba, PR, CEP: 80215-901 


\section{INTRODUÇÃO}

A Insuficiência Cardíaca (IC) é uma síndrome multifatorial com abrangência sistêmica, caracterizada pela disfunção cardíaca, com disfunção ventricular esquerda sistólica em $60 \%$ dos casos. Essa síndrome resulta em importantes alterações hemodinâmicas, tais como inadequado débito cardíaco e elevação da pressão pulmonar e venosa sistêmica. 0 débito cardíaco reduzido leva a uma perfusão tecidual insuficiente, com manifestações durante o exercício na fase inicial da doença, e progressão com sintomas em repouso em uma fase mais avançada ${ }^{1}$.

A IC tem elevada prevalência entre os idosos, com $75 \%$ dos doentes acima de 65 anos. 0 envelhecimento, intrinsicamente, predispõe a um aumento da sua incidência, bem como da morbidade e mortalidade decorrente, de forma que IC é a principal causa de hospitalização senil ${ }^{2-4}$.

Os principais fatores de risco para o seu desenvolvimento incluem: sexo masculino, baixo nível educacional, sedentarismo, tabagismo, obesidade, diabetes mellitus, hipertensão arterial (HAS), doença valvar do coração, hipertrofia de ventrículo esquerdo e doenças coronarianas $^{3}$. Diversas comorbidades podem desencadear descompensação da IC, incluindo doenças tireoidianas, anemia, insuficiência renal e apneia do sono, contudo a miocardiopatia isquêmica nessa parcela da população é a principal etiologia da disfunção sistólica4.

Essa doença é caracterizada por alterações em muitos mecanismos neuro-hormonais, mais notadamente do sistema simpático e do renina-angiotensinaaldosterona. Sendo assim, a inibição ou modulação desses dois sistemas é a base do tratamento5. A terapia "standart" recomendada para pacientes com IC, segundo a American College of Cardiology (ACC) e American Heart Association (AHA), é a associação de um inibidor da enzima conversora da angiotensina II (iECA) ou bloqueador do receptor de angiotensina II (BRA) com betabloqueadores (BB). Essa associação medicamentosa está associada a menores taxas de mortalidade e readmissão hospitalar6.

Os beta-bloqueadores por si, são considerados drogas disruptivas nessa doença, tendo em vista que, em pacientes com IC sistólica, oferecem benefícios clínicos comprovadoscomo redução da progressão dos sintomas e da própria disfunção de VE, além de redução da internação hospitalar e da mortalidade ${ }^{1}$.

Mesmo tais drogas tendo sido extenuantemente avaliadas no contextolC, os grandes estudos clínicos em geral excluem os idosos, especialmente devido à presença de inúmeras comorbidades, aumento de efeitos adversos, presença de polifarmácia, interação medicamentosa e alterações típicas do organismo em envelhecimento. Esses fatores levam à dificuldade de interpretação e extrapolação dos resultados e do benefício do tratamento com beta-bloqueadores nessa população. Sabe-se por diversos registros que a terapêutica para IC, em função da lacuna de informações, tem sido prescrita de maneira inadequada e em muitos casos subprescrita? ${ }^{7}$.

As diretrizes ACC/AHA IC recomendam como nivel de evidência classe I, que pacientes idosos, mesmo que sub-representados nos ensaios clínicos e na ausência de provas específicas para tratar de outro modo, devem receber triagem clínica e terapêutica semelhante à prevista para a população geral8.

O objetivo desta pesquisa é apresentar uma revisão de literatura sobre o uso de beta-bloqueadores em pacientes idosos com insuficiência cardíaca sistólica.

\section{MÉTODOS}

O desenvolvimento desta revisão de literatura foi realizado por meio de busca em bases de dados eletrônicas, tais como: Lilacs, Scielo, PubMed, Google Acadêmico, MedLine, UpToDate, Biblioteca Virtual da Saúde e MedScape. Considerando os períodos de agosto de 2014 a janeiro de 2015, incluindo os idiomas português e inglês. Os descritores utilizados para a busca das pesquisas foram insuficiência cardíaca, insuficiência cardíaca sistólica, tratamento da IC sistólica, betabloqueadores na IC sistólica, beta-bloqueadores na IC sistólica em idosos maiores de 65 anos.

Para a seleção dos artigos foram utilizados critérios de inclusão selecionados juntamente com a orientadora, tais como: resultados de pesquisa de acordo com a relevância relacionada ao título e aos objetivos da revisão, baseados na leitura dos seus respectivos resumos, restringindo-se aos beta-bloqueadores na IC sistólica em idosos maiores que 65 anos. Realizou-se uma leitura crítica de cada artigo selecionado e em seguida foram realizadas comparações das informações de cada um deles.

Para análise foram selecionados 17 artigos, sendo oito provenientes de países da América do Norte, sete da Europa e dois nacionais. Consideraram-se artigos não extraídos das bases citadas, mas pertinentes ao objetivo da revisão porque atendem a um dos critérios de inclusão acima citados: Bocchi et al (2009); e Brookes (2005).

A apresentação e discussão dos resultados encontrados serão apresentadas sob quatro perspectivas analíticas: a Insuficiência Cardíaca, o uso de betabloqueadores na IC, sua eficácia terapêutica e a sua prescrição em idosos.

\section{REVISÃO DE LITERATURA}

\section{Insuficiência cardíaca}

A IC é uma enfermidade em progressão, o envelhecimento da população e a maior sobrevida em pacientes cardíacos são os principais contribuintes. Ainda 
assim, a mortalidade é muito elevada. A prevalência é semelhante entre os gênerose maior em afro americanos quando comparada aos caucasianos ${ }^{9}$.

No Brasil, representa um importante impacto à saúde pública. No período de 2000 a 2007 houve um aumento dos gastos decorrentes de hospitalização em $11,3 \%$. No ano de 2007 as doenças cardiovasculares representaram a 3a causa de internação no SUS, sendo a IC a mais frequente. A cardiopatia isquêmica crônica associada à HAS é a principal etiologia dessa doença ${ }^{1}$.

A classificação mais utilizada é a proposta pela New York Heart Association (NYHA) que estratifica em quatro classes baseadas na intensidade dos sintomas (Classe I-IV), já a evolução da doença é dividida em quatro estágios (A-D) determinando qualidade de vida, prognóstico e condutas terapêuticas ${ }^{1}$.

A taxa de mortalidade intra-hospitalar varia conforme a faixa etária, sendo maior nos idosos e nos pacientes menores de 20 anos $^{1}$. A presença de múltiplas comorbidades com o avançar da idade influenciam na evolução e prognóstico da doença, e dificultam o tratamento que deverá ser individualizado nesses pacientes.

O envelhecimento causa alterações cardíacas progressivas estruturais e funcionais levando ao aumento da vulnerabilidade. 0 enrijecimento arterial determinado ao longo dos anos e redução da complacência arterial ocasiona a elevação da pressão arterial sistólica (PAS), HAS, hipertrofia ventricular, aumento atrial e comprometimento da adaptação às situações de sobrecarga 4 .

Lesões endoteliais progressivas, com redução da capacidade de vasodilatação, associadas a alteração no metabolismo lipoproteico contribuem para formação de placas ateroscleróticas. A dessensibilização dos receptores beta-adrenérgicos por sua vez, provocou um aumento da pós carga e uma diminuição da resposta cronotrópica e inotrópica. Além disso, a frequência cardíaca máxima reduzida influencia na diminuição da capacidade física e está relacionada a maior morbimortalidade nos idosos 4 .

\section{B-bloqueadores na Insuficiência Cardíaca}

A IC sistólica é caracterizada por alterações em diversos mecanismos neuro-hormonais e um aumento na atividade simpática, apresentando cronicamente efeitos deletérios a função e geometria ventricular. Os BB são drogas de $1^{a}$ linha no tratamento devido ao seu antagonismo no sistema simpático, levando à melhora clínica e da função ventricular, com aumento da sobrevida nos pacientes ${ }^{1,10}$.

A estimulação crônica dos receptores beta ajuda a restaurar a capacidade de resposta inotrópica e cronotrópica do miocárdio, melhorando a sua função contrátil. Essas drogas também reduzem o nível circulante de vasoconstritores, como noradrenalina, renina e endotelina, diminuindo a progressão da disfunção cardíaca; atuam no remodelamento reverso do ventrículo esquerdo (VE); reduzem as citocinas inflamatórias, os volumes sistólico e diastólico finais; e melhoram a perfusão na miocardiopatia isquêmica ${ }^{10}$.

0 uso de bloqueadores de receptores beta adrenérgicos é contraindicado em pacientes com doenças reativas das vias aéreas, IC descompensada, choque cardiogênico, bloqueio cardíaco de segundo ou terceiro grau e síndrome do nó sinusal11. Deve haver monitorização durante o seu uso, devido aos riscos de hipotensão, bradicardia e piora da insuficiência cardíaca ${ }^{12}$.

Os clinicamente comprovados no tratamento da IC são: carvedilol, bisoprolol, succinato de metoprolol e mais recentemente o nebivolol, os quais apresentam seletividade em receptores beta 1 adrenérgicos ${ }^{1}$.

o bisoprolol e o nebivolol possuem maior seletividade, o succinato de metoprolol vem na sequência. Esses três medicamentos apresentam menor ação lipofílica, com menor atuação sobre o SNC e sobre o metabolismo hepático, o que possibilita a administração posológica de uma vez ao dia. Já o carvedilol possui maior ação no SNC, menor meia-vida e pode causar remodelamento reverso da função simpática cardíaca1.

\section{Eficácia terapêutica}

0 estudo CIBIS avaliou o uso de betabloqueadores em pacientes com insuficiência cardíaca crônica, entre 18 e 75 anos, em classe funcional III e IV da NYHA, fração de ejeção menor ou igual a $40 \%$, com estabilidade clínica e em uso de terapia vasodilatadora associada a um diurético. Este foi o primeiro estudo em grande escala a testar os betabloqueadores na ICC, tendo como desfecho a mortalidade, porém, não constatou redução significante no grupo de pacientes em uso de bisoprolol (16,6\%) em relação ao grupo do placebo (20,9\%).

Em relação a etiologia da IC, observou-se maior redução na mortalidade (50\%) no grupo em uso de bisoprolol em pacientes sem história de IAM e cardiomiopatia dilatada idiopática. No entanto, não houve explicação clara para a diferença dos resultados em relação a etiologia.

Concluiu-se que os pacientes apresentaram melhora da classe funcional NYHA e menor necessidade de hospitalização por descompensação da IC. Verificou-se também que o aumento progressivo da dose dos BB levou a uma melhora funcional e hemodinâmica nos pacientes ${ }^{13}$.

Já o estudo CIBIS II incluiu pacientes entre $18 \mathrm{e}$ 80 anos de idade, em classe funcional III e IV da NYHA, fração de ejeção menor ou igual a $35 \%$, em uso de iECA associado a diurético. Obteve um risco 32\% menor para mortalidade e hospitalização para IC, em pacientes em uso de bisoprolol em comparação ao placebo, independente da 
etiologia ou classe funcional. Essa diferença significante levou a interrupção precoce do ensaio. Houve maior benefício para pacientes com doença isquêmica do miocárdio, em classe funcional III NYHA no início do estudo, contrastando com os achados do estudo CIBIS.

0 efeito beta-1 seletivo dos BB mostrou-se suficiente em reduzir as taxas de morte súbita, em relação aos não seletivos.0 bisoprolol foi mais efetivo em prevenir a morte súbita em relação a morte por falência de bomba, sugerindo maior efeito antiarrítmico desse medicamento.

Recomendou-se a administração gradual e progressiva dos $\mathrm{BB}$, iniciando com doses baixas $\mathrm{e}$ atingindo a máxima tolerada. 0 estudo ressaltou ainda a falta de dados sobre o efeito do tratamento em idosos 14 .

O estudo CIBIS III, que analisou pacientes > 65 anos com IC de reduzida fração de ejeção e classe funcional II e III, avaliou a presença de alterações na morbidade e mortalidade segundo a ordem de início dos principais medicamentos preconizados para tratamento. Constatou-se que o uso de monoterapia inicial com bisoprolol apresentou igual eficácia terapêutica quando comparada à iniciada com maleato de enalapril. Verificouse ainda menor risco de morte súbita com o uso de bisoprolol em comparação ao enalapril, o que revelou a tendência para melhora da sobrevida nesse grupo, especialmente no primeiro ano13. Não houve diferença significante entre os grupos em relação à hospitalização ou mortalidade por qualquer causa.

Já se sabe que os BB podem causar um pequeno agravamento temporário dos sintomas de IC, o que foi mais evidenciado no grupo do bisoprolol. Nesse contexto, o estudo levantou a hipótese de que o início da terapia sem um iECA prévio, pode levar a implicações diferentes sobre seu efeito inotrópico negativo. Além disso, os médicos tem experiência limitada com a titulação da dose de BB em tratamentos não precedidos pelo iECA.

Constatou-se ainda, índices semelhantes de efeitos adversos e dos níveis pressóricos em ambos os grupos. Houve uma redução mais evidenciada da frequência cardíaca no primeiro ano nos pacientes tratados em monoterapia com bisoprolol, porém os valores se tornaram equivalentes desde então.

O estudo concluiu que os médicos possuem a liberdade de iniciar o tratamento da IC com uma ou outra classe de drogas, diferindo do recomendado pelas diretrizes, como também uma titulação lenta dos BB para a dose máxima permitida dentro dos níveis alvo tem uso seguro e bem tolerado em idosos e portadores de comorbidades ${ }^{15}$.

Outro estudo comparou o uso de carvedilol versus placebo em pacientes com IC de moderada a grave intensidade e fração de ejeção menor ou igual a 35\%, em uso de iECA, digoxina e diurético. Os pacientes em uso de carvedilol obtiveram maior frequência de melhora sintomática e menor risco de deterioração clínica comparados com o placebo, entretanto com pouco efeito na melhora do exercício físico. Além disso, houve um aumento significativo da fração de ejeção e uma redução importante no risco de morbidade e mortalidade no grupo do carvedilol. Os efeitos foram semelhantes na doença de etiologia isquêmica ou cardiomiopatia dilatada idiopática 16.

O estudo SENIORS mostra que o tratamento de pacientes idosos > 70 anos com IC, em uso de nebivolol, é bem tolerado e eficaz na redução da morbimortalidade, independentemente da fração de ejeção inicial. Atua também na redução da mortalidade e admissão hospitalar por qualquer causa cardiovascular em comparação ao placebo. Os efeitos benéficos surgem após seis meses de tratamento e continuam a aumentar com o tempo ${ }^{5}$.

A medicação para o estudo foi bem tolerada e a maioria dos pacientes foram capazes de chegar a uma dose de manutenção de $10 \mathrm{mg}$ uma vez por dia. Ainda que os pacientes idosos tenham maior risco de efeitos adversos, como hipotensão e bradicardia, em relação aos pacientes mais jovens, $68 \%$ conseguiram atingir a dose máxima, e somente $6 \%$ não suportaram qualquer dose. Essa boa tolerabilidade pode estar relacionada, em parte, com a propriedade vasodilatadora do nebivolol, não podendo ser, portanto, generalizada para outros BB quando utilizados em pacientes idosos com IC.

Embora os benefícios do nebivolol pareçam ser menores em pacientes > 75 anos, a idade como uma variável contínua não afetou, significativamente, o efeito do tratamento. É possível que os beta-bloqueadores, ou mesmo todos os tratamentos, sejam na realidade, menos eficazes no paciente muito idoso.

A interrupção prematura do tratamento ocorreu em $27 \%$ dos casos que utilizaram o nebivolol, e $25 \%$ no grupo placebo. A principal causa da suspensão foi a pedido dos pacientes e outras razões não clínicas, com exceção do óbito. 0 que evidenciou pouca diferença entre os dois grupos 5 .

\section{Prescrição em idosos}

Os dados dos guidelines demonstraram que as terapias recomendadas são frequentemente usadas em pacientes idosos, incluindo aqueles com > 85 anos. Porém, diferenças na terapia relacionadas à idade persistem, sugerindo que as oportunidades para melhorar o atendimento ainda permanecem. Otimizar o tratamento da IC nesses pacientes é uma prioridade crescente na área da saúde ${ }^{2}$.

O aumento da idade foi um preditor significativo de menor taxa de adesão para as terapias. Os guidelines preconizam que o tratamento da IC sistólica deve ser o mesmo para pacientes idosos e não idosos. Apesar dessa parcela populacional estar em maior risco de morbidade e mortalidade, foi constatado um declínio da prescrição de BB em relação aos pacientes mais jovens 8,17 . Nesse contexto, não foi verificado diferença no benefício de tratamento entre idosos e pacientes mais jovens, nos 
estudos MERIT-HF e Meta Analysis of $>12,000$ Patients in Larg-Scale Trials ${ }^{11}$.

Os pacientes menos propensos a receber tratamento com BB foram os do sexo feminino, moradores em áreas de privação e portadores de DPOC, mesmo este não estando entre as contraindicações. Os portadores de doença isquêmica cardíaca, HAS e fibrilação atrial foram os que mais receberam ${ }^{17}$.

Em relação à prescrição esperada, estudos verificaram aumento na taxa de uso dos betabloqueadores 6,17 . Porém, um viés desse dado foi a possível coleta de informações ambulatoriais e na alta hospitalar ${ }^{17}$, não sabendo sobre a continuidade do uso. Outra limitação foi a consciência dos médicos a respeito do estudo2. Esses resultados diferem dos encontrados anteriormente, os quais mostraram que esses medicamentos são subutilizados e que deveriam ser criadas estratégias para aumentar o seu uso18-20.

Pacientes em classe funcional III e IV (NYHA) receberam doses menores de $\mathrm{BB}$ em relação às preconizadas, assim como os portadores de doença de Chagas. Já os com cardiopatia isquêmica, receberam a dose-alvo ou maior. A dose encontrada nesse estudo foi bem acima das doses relatadas em registros e ensaios clínicos.

Foi demonstrado que a maioria dos pacientes pode atingir e tolerar a dose-alvo das diretrizes e que doses mais elevadas estão associadas a melhores resultados, como maior reversão da dilatação cardíaca e diminuição da morbimortalidade 21 .

A razão mais citada para a menor prescrição pelos médicos é a falta de evidências para essa faixa etária5. Além disso, há incerteza sobre os riscos e vantagens, percepções errôneas a respeito das contraindicações, falta de tolerância nessa população, polifarmácia, desejo de evitar os efeitos adversos e interações medicamentosas ${ }^{8}$.

A subutilização dessa classe medicamentosa em alguns casos pode ser atribuída à questão financeira e ao uso prolongado dessa medicação ${ }^{22}$. Essas variações terapêuticas podem refletir também questões como a qualidade de atendimento, as preferências do paciente e outras considerações não médicas, como acesso aos cuidados e outras comorbidades 8 .

\section{CONCLUSÃO}

Os beta-bloqueadores apresentam benefício clínico na redução da morbimortalidade. Estudos comprovaram que idosos toleram a dose-alvo, e que doses elevadas e tempo prolongado de tratamento relacionam-se a melhores resultados.

Verificou-se uma divergência presente na literatura, principalmente em relação as taxas de uso e de prescrição, o que pode ser um importante fator contribuinte na insegurança dos médicos com essa população.

A lacuna de grandes estudos clínicos sobre esse tema é a causa preponderante de prescrição inadequada e subprescrição, evidenciando a necessidade de maiores pesquisas. Esses fatores associados aos limites das diretrizes levam a necessidade de terapia individualizada.

\section{REFERÊNCIAS}

1. Andrade JP De, Siciliano A, David J, Neto DS, Moreira LF, Teixeira RA, et al. III Diretriz Brasileira de Insuficiência Cardíaca Crônica. 2009.

2. 2. Forman DE, Cannon CP, Hernandez AF, Liang L, Yancy C, Fonarow GC. Influence of Age on the Management of Heart Failure : Findings from Get With the Guidelines - Heart Failure. Am Hear J. 2009; (January 2005):1-13.

3. 3. Jessica C, A. SS. Assessment and Management of Heart Disease Related to Complex Care of Older Adults. 2007.

4. 4. Wajngarten M. O Coração do Idoso. J Diagnósticos em Cardiol. 2010;1-9.

5. 5. Flather MD, Shibata MC, Coats AJS, Van Veldhuisen DJ, Parkhomenko A, Borbola J, et al. Randomized trial to determine the effect of nebivolol on mortality and cardiovascular hospital admission in elderly patients with heart failure (SENIORS). Eur Heart J [Internet]. 2005 Feb [cited 2015 May 7];26(3):215-25. Available from: http://www.ncbi.nlm.nih.gov/pubmed/15642700

6. 6. Dimartino LD, Shea AM, Hernandez AF, Lesley $H$. Use of Guideline-Recomended Therapie for Heart Failure in the Medicare Population. Clin Cardiol. 2010;33(7):400-5.

7. 7. Rochon PA. Drug prescribing for older adults. Wolters Kluwer Heal - Up To Date. 2014;1-31.

8. 8. Yancy CW, Fonarow GC, Albert NM, Curtis AB, Stough WG, Gheorghiade M, et al. Influence of patient age and sex on delivery of guideline-recommended heart failure care in the outpatient cardiology practice setting: findings from IMPROVE HF. Am Heart J [Internet]. Mosby, Inc.; 2009 Apr [cited 2015 May 7];157(4):754-62.e2. Available from: http://www.ncbi.nlm.nih.gov/pubmed/19332206

9. 9. Vasan RS, Wilson PWF. Epidemiology and causes of heart failure. Wolters Kluwer Heal - Up To Date. 2015;1-15.

10. 10. Colucci WS. Rationale for and clinical trials of beta blockers in heart failure due to systolic disfunction. Wolters Kluwer Heal - Up To Date. 2014;1-28.

11. 11. Man JP, Jugdutt BI. Systolic heart failure in the elderly: optimizing medical management. Hear Fail Rev [Internet]. 2012 Sep [cited 2015 May 7];17(4-5):563-71. Available from: http://www.ncbi.nlm.nih.gov/pubmed/22002260

12. 12 . O'Connor CM, Arumugham P. Inotropic drugs and neurohormonal antagonists in the treatment of HF in the elderly. Clin Geriatr Med 
[Internet]. 2007 Feb [cited 2015 Apr 2];23(1):141-

53.

Available

from:

http://www.ncbi.nlm.nih.gov/pubmed/17126759

13. 13. CIBIS Investigators and Committees. A randomized trial of beta-blockade in heart failure. The Cardiac Insufficiency Bisoprolol Study (CIBIS). Circulation [Internet]. 1994;90(4):1765-73. Available from: http://www.ncbi.nlm.nih.gov/pubmed/7923660

14. 14.Dargie HJ, Lechat P. The Cardiac Insufficiency Bisoprolol Study II (CIBIS-II): A randomised trial. Lancet. 1999;353(9146):9-13

15. $15 . \quad B r o o k e s$ L. CIBIS III: Cardiac Insuffiency Bisoprolol Study III. Medscape. 2005;Oct 4:1-2.

16. 16. Packer M, Colucci WS, Sacknerbernstein JD, Liang CS, Goldscher D a, Freeman I, et al. Double blind, placebo controlled study of the effects of carvedilol in patients with moderate to severe heart failure - the precise trial. Circulation. 1996;94:27939.

17. $17 . \quad$ Shah SM, Carey IM, DeWilde S, Richards N, Cook DG. Trends and inequities in beta-blocker prescribing for heart failure. $\mathrm{Br} J$ Gen Pract [Internet]. 2008 Dec [cited 2015 May 7];58(557):862-9. Available from: http://www.pubmedcentral.nih.gov/articlerender.fcgi ?artid=2593536\&tool=pmcentrez\&rendertype=abstr act

18. 18. Ko DT, Tu K V., Masoudi FA, Wang Y. Quality of Care and Outcomes of Older Patients With Heart Failure Hospitalized in the United States and Canada. Arch Intern Med. 2005;165:2486-92.

19. 19. Muntwyler J, Cohen-Solal A, Freemantle N, Eastaugh J, Cleland JG, Follath F. Relation of sex, age and concomitant diseases to drug prescription for heart failure in primary care in Europe. Eur J Heart Fail [Internet]. 2004 Aug [cited 2015 May 2];6(5):663-8. Available from: http://www.ncbi.nlm.nih.gov/pubmed/15302016

20. 20. Smith NL, Chan JD, Rea TD, Wiggins KL, Gottdiener JS, Lumley T, et al. Time trends in the use of beta-blockers and other pharmacotherapies in older adults with congestive heart failure. Am Heart J [Internet]. 2004 Oct [cited 2015 May 8];148(4):7107. Available from: http://www.ncbi.nlm.nih.gov/pubmed/15459605

21. 21. Moreno IB, Carlo CH Del, PereiraBarretto AC. Tratamento Otimizado e Redução da Frequência Cardíaca na Insuficiência Cardíaca Crônica. Arq Bras Cardiol [Internet]. 2013 Nov [cited 2015 May 7];101(5):442-8. Available from: http://www.pubmedcentral.nih.gov/articlerender.fcgi ?artid $=4081168 \&$ tool $=$ pmcentrez\&rendertype $=$ abstr act

22. 22. Donohue JM, Ph D, Zhang Y, Lave JR, Gellad WF, Men A, et al. The Medicare Drug Benefit (Part D) and Treatment of Heart Failure in Older Adults. Am Hear J. 2011;160(1):159-65. 